\title{
Ultrafiltration biofeedback guided by blood volume monitoring to reduce intradialytic hypotensive episodes in hemodialysis: study protocol for a randomized controlled trial
}

\author{
Kelvin CW Leung ${ }^{1 *}$, Robert R Quinn ${ }^{1,2}$, Pietro Ravani ${ }^{1,2}$ and Jennifer M MacRae
}

\begin{abstract}
Background: Fluid removal during dialysis, also known as ultrafiltration (UF), leads to intradialytic hypotension (IDH) in a significant number of patients treated with hemodialysis (HD) and is associated with an increase in morbidity and mortality. At present, there are no accepted standards of practice for the prevention or treatment of IDH. Relative blood volume monitoring (BVM) is based on the concept that the hematocrit increases with UF, relative to the patient's baseline hematocrit. The use of BVM biofeedback, whereby the HD machine automatically adjusts the rate of UF based on the relative blood volume, has been proposed for the prevention of IDH.

Methods/Design: This is a 22-week randomized crossover trial. Participants undergo a 4-week run-in phase to standardize medications and dialysis prescriptions. Subsequently, participants are randomized to standard HD or to BVM biofeedback for a period of 8 weeks followed by a 2-week washout phase before crossing over. The dialysis prescription remains identical for both arms. The primary outcome is the frequency of symptomatic IDH as defined by an abrupt drop in the systolic blood pressure of $\geq 20 \mathrm{~mm} \mathrm{Hg}$ accompanied by headache, dizziness, loss of consciousness, thirst, dyspnea, angina, muscle cramps or vomiting. Secondary outcomes include the number of symptomatic IDH episodes and any reduction in IDH episodes, nursing interventions, dialysis adequacy, total body water, extra- and intracellular fluid volumes, brain natriuretic peptide and cardiac troponin levels, blood pressure, antihypertensive medication use, patient symptoms and quality of life.
\end{abstract}

Discussion: Our study will determine the impact of using BVM biofeedback to prevent IDH and other serious adverse events in susceptible patients.

Trial registration: Clinicaltrials.gov NCT01988181 (6 November 2013).

Keywords: Hemodialysis, Blood volume monitoring biofeedback, Intradialytic hypotension, Randomized crossover trial

\section{Background}

The majority of patients with end-stage renal disease are treated with hemodialysis (HD) to regulate their fluid balance and other native kidney functions [1,2]. Patients treated with HD have a 5-year mortality rate of $57 \%$, with the majority of deaths due to cardiac and infectious causes [3,4]. A growing body of literature has emerged linking chronic fluid overload in HD to hypertension, left ventricular hypertrophy and increased all-cause

\footnotetext{
* Correspondence: kcwleung@ucalgary.ca

'Department of Medicine, University of Calgary, Calgary, Canada

Full list of author information is available at the end of the article
}

mortality [5-7]. To prevent volume overload and maintain fluid balance, 1 to $5 \mathrm{~L}$ of fluid must be removed during each HD treatment.

Rapid fluid removal, or ultrafiltration (UF), during a short period of time can lead to intradialytic hypotension (IDH) in as many as $25 \%$ to $50 \%$ of patients treated with HD [8]. IDH is most commonly defined as an abrupt drop in systolic blood pressure of $\geq 20 \mathrm{~mm} \mathrm{Hg}$ accompanied by symptoms of cerebral, cardiac, gastrointestinal or musculoskeletal ischemia [9-11]. Observational studies have 
shown that IDH or a drop in post-dialysis systolic blood pressure is associated with an increase in morbidity and mortality [12-20].

UF during HD leads to a fall in the patient's blood volume. The blood volume can be measured by tracking the changes in hemoglobin or protein concentration at the arterial port during dialysis using optical photometry or ultrasound [21-28]. In clinical studies, higher rates of UF lead to faster declines in the relative blood volume and as a result, a steeper decline in the blood volume monitoring (BVM) curves [27-29]. BVM-guided biofeedback is based on the principle that the relative blood volume (compared to baseline) appears to decrease more rapidly in sessions complicated by IDH $[25,30]$. As a result, BVM-guided UF biofeedback, whereby the dialysis machine automatically reduces the rate of UF prior to reaching the patient's critical blood volume, has been proposed for the prevention of IDH and its sequelae [22,23,31-34].

We will conduct a randomized controlled clinical trial to test whether biofeedback incorporating BVM-guided UF adjustments alone (without adjustment of the dialysate sodium concentration), in addition to best clinical practice, results in a reduction in the frequency of symptomatic IDH episodes and patient symptoms compared to best clinical practice alone. Secondary outcomes include the frequency of IDH-related interventions, dialysis-related symptoms, dialysis adequacy, volume control, biomarkers of volume overload and cardiac stress, blood pressure and quality of life.

\section{Methods/Design}

\section{Study design}

This is a 22-week parallel-group randomized crossover trial. During the first part of the study, the run-in and dialysis optimization phase, eligible participants will undergo a 4-week run-in. During this period, all participants will undergo a comprehensive clinical assessment including a clinical weight assessment, an antihypertensive medication review and standardization of their dialysis prescription. At the end of the run-in phase, participants that still meet the eligibility criteria will enter part 2, the randomized crossover phase. In this phase, the participants are randomized to regular best clinical practice HD (without BVM-guided UF biofeedback; the control arm) or to best clinical practice plus BVM-guided UF biofeedback (the intervention arm) for an 8 -week period. This will be followed by a 2 -week washout phase and then the participants will be crossed over for a second 8-week phase. The participant flow chart and timeline are shown in Figure 1. The study will be conducted and reported following the Consolidated Standards of Reporting Trials (CONSORT) 2010 guidelines [35]. The study protocol was approved by the University of Calgary Conjoint Health Research Ethics Board (ethics ID: REB13-1139).

\section{Study setting}

The clinical trial will be held at two tertiary care units (Foothills Medical Centre and Peter Lougheed Centre) and three community HD units (Fanning Centre, Sheldon Chumir Centre and Sunridge Centre) in the Southern Alberta Renal Program, Calgary, Alberta, Canada.

\section{Study participants}

All participants who have been treated with HD for more than 3 months will be screened for eligibility. To be eligible for the study, participants must be $>18$ years of age, medically stable, undergo HD three to four times per week for a minimum of 3 hours per session, and have had $>30 \%$ of their HD sessions in the preceding 8 weeks complicated by symptomatic IDH. Participants with serum sodium $\leq 133 \mathrm{mmol} / \mathrm{L}$ [36], hemoglobin $<80 \mathrm{~g} / \mathrm{L}$, active malignancy, a history of blood transfusion or hospitalization in the preceding 4 weeks, routine use of diuretics for volume management, a history of ongoing urine output estimated at greater than or equal to $250 \mathrm{~mL}$ (one cup) per day, or a planned change in the renal replacement modality during the study period will be excluded. Informed consent will be obtained from each individual who agrees to participate in the study.

\section{Definition of symptomatic intradialytic hypotension}

Symptomatic IDH is defined as a drop in systolic blood pressure of $\geq 20 \mathrm{~mm} \mathrm{Hg}$ from baseline with associated symptoms $[9,10]$. Symptoms include sudden-onset headache, dizziness, unconsciousness, thirst, dyspnea, angina, muscle cramps and vomiting $[10,11]$.

\section{Interventions}

\section{Part 1: run-in phase}

Following enrollment, participants will undergo a 4-week run-in phase to optimize their dialysis weight and dialysis prescriptions, and to determine the critical relative blood volume values (BVM will be enabled). During the first 2 weeks of the run-in phase, participants will undergo a medication review and dry-weight reduction based on a modified protocol from the Dry-Weight Reduction In Hypertensive Hemodialysis Patient (DRIP) trial $[37,38]$.

\section{Part 2: crossover study phase}

Participants that continue to have more than $30 \%$ of their sessions complicated by symptomatic IDH during the run-in phase are randomized to either 8 weeks of best clinical practice (without BVM or biofeedback; control) or best clinical practice plus BVM-guided UF biofeedback (intervention), followed by a 2 -week washout period (using the control phase HD prescription) before crossing over to the other study arm for a second 8-week block. Participants will have clinical assessments of their dry weight at the beginning of each week. 


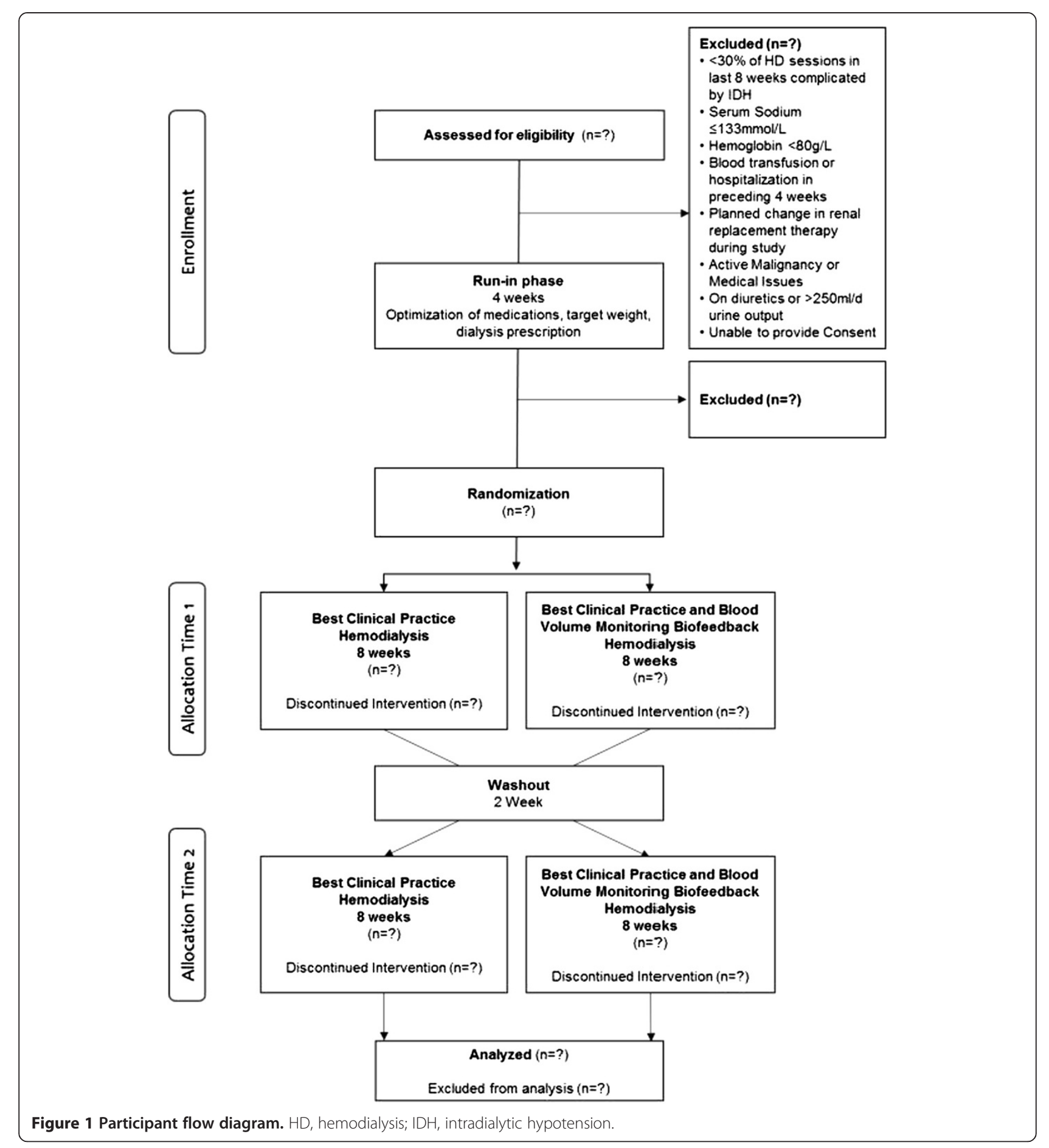

\section{Control group}

All study participants will be dialyzed with the Fresenius 5008 HD machine (Fresenius Medical Care, Bad Homburg, Germany) using high-flux dialyzers. Participants in the best clinical practice (control) group will use the same prescription used during the run-in phase: dialysate sodium of $138 \mathrm{mmol} / \mathrm{L}$, dialysate calcium of $1.25 \mathrm{mmol} / \mathrm{L}$, dialysate temperature of $36^{\circ} \mathrm{C}$ and constant UF rate. BVM will be disabled in this group.

\section{Intervention group}

Participants in the BVM-guided UF biofeedback group will have the same prescription as the control group, but will also have the UF rate automatically adjusted by the 
Fresenius 5008 HD machine based on changes in relative blood volume.

\section{Ultrafiltration biofeedback guided by blood volume monitoring using the Fresenius $\mathbf{5 0 0 8}$}

The Fresenius 5008 uses an ultrasound monitor incorporated into the machine to detect ultrasonic velocity changes to derive the total protein concentration, which is a sum of total plasma proteins and hemoglobin. A temperature monitor is also incorporated to correct for temperature-related changes in ultrasound velocity [21,39]. Since the total protein does not change, any changes in its concentration are attributed to blood volume changes (Figure 2). The relative blood volume is calculated by dividing the initial concentration of total protein by the total protein concentration at any given time, multiplied by 100 [21]. This method for measuring relative blood volume has been previously validated with both optical and laboratory hemoglobin techniques [21,24].

The HD software in the Fresenius 5008 HD machine adjusts the UF rate based on the critical blood volume entered at the beginning of the dialysis session for each individual patient. There is no adjustment to the dialysate sodium concentration. To determine the actual UF rate, the HD software first calculates the maximum UF rate. The maximum UF rate is 2 times the total UF divided by the remaining time. The actual UF rate is the maximum UF rate multiplied by the UF coefficient, which is a number between zero and one. To allow for the maximum UF at the onset of HD, the UF coefficient is one at the start of $\mathrm{HD}$ and continues to remain at one until the relative blood volume is halfway towards the critical relative blood volume. At the halfway point between the start and the critical relative blood volume, the UF coefficient decreases linearly. Once the critical relative blood volume is reached, the UF coefficient becomes zero, resulting in cessation of UF $[21,24,40]$.

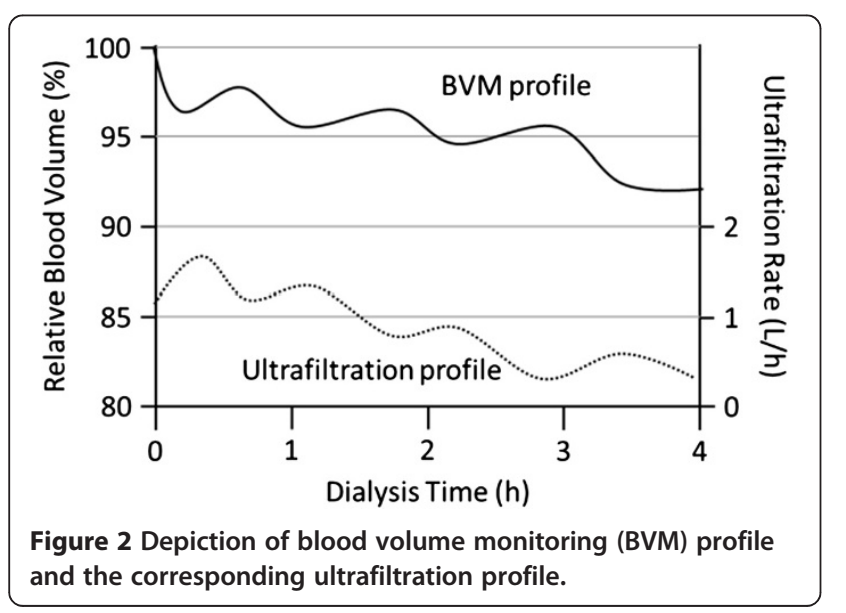

\section{Determination of critical blood volume}

There is currently no standardized method for determining the critical relative blood volume. To standardize this process, a single investigator (KL) will use the following method/algorithm:

1. Identify the most recent episode of symptomatic IDH as per the study definition.

2. The critical blood volume will be equal to the relative blood volume recorded immediately prior to the episode of symptomatic IDH.

3. The critical blood volume will be reassessed weekly.

\section{Weight adjustments}

Each week participants will have their target weight assessed by a single study investigator (KL). The rounding dialysis physician will be encouraged to discuss any weight adjustments with study personnel.

\section{Responding to episodes of intradialytic hypotension}

In the event that an IDH episode occurs, the bedside dialysis nurse will follow a predefined IDH algorithm (Additional file 1) [38]. Following resolution of an IDH episode as defined in the primary outcome section, UF will resume. In the control group, a constant UF rate will be reset to meet the UF goal. In the intervention group, BVM-guided UF biofeedback will be re-enabled.

\section{Outcomes}

\section{Primary outcome}

The primary outcome is the rate of symptomatic IDH. The number of symptomatic IDH episodes along with the duration of each dialysis treatment will be captured. The rate of IDH for each session will be calculated by dividing the number of episodes by the duration of the session in hours. The rate of IDH will be calculated for every dialysis treatment. The rate of symptomatic IDH will be measured in the 2 months preceding enrolment and during each phase of the study. By using the rate of symptomatic IDH, rather than the frequency or number of IDH episodes per session as done in previous studies, we will be more sensitive to meaningful changes in IDH episodes as multiple episodes of symptomatic IDH can occur over various durations of dialysis $[31,32,41]$.

\section{Secondary outcomes}

The secondary outcomes of interest are the number and frequency of nursing interventions, the number and frequency of both symptomatic and asymptomatic IDH, the number and frequency of dialysis symptoms, dialysis adequacy as measured by single-session $K t / V$, total body water, extracellular fluid volume (ECFV), intracellular fluid volume (ICFV), the ECFV:ICFV ratio as determined by electrical bioimpedance, changes in brain natriuretic 
peptide (BNP) level, changes in high-sensitivity-cardiac troponin level, changes in blood pressure (mean arterial pressure pre-, intra- and post-dialysis), changes in antihypertensive medications, changes in the BVM curves, intradialytic and interdialytic symptom survey, and nursing satisfaction.

IDH-related nursing interventions are defined as the use of the Trendelenburg/supine position, discontinuation of UF, fluid/saline challenge, return of blood, shortened dialysis run, or a prolonged (more than 15 minutes) post-dialysis recovery period as a result of IDH or its symptoms. Given the subjective nature of IDH-related symptoms and intervention, in-services and customized dialysis run sheets will be provided to all nurses at participating dialysis centers to ensure that all events are recorded, and recorded consistently.

Dialysis adequacy, measured by single-pool $K t / V$, will be measured and recorded (usual care) at the end of each dialysis session on the session sheet. Single-pool $K t / V$ will be calculated using the previously validated, online clearance measurement method, which detects changes in conductivity in the dialysate to reflect the clearance of serum electrolytes and urea [42].

Whole body and segmental bioimpedance analysis has been validated for the determination of fluid composition in HD patients [43-47]. Electrical bioimpedance will be performed during the mid-week HD session of weeks $1,4,8,12,14,18$ and 22 of the study to determine the total body water, ECFV and ICFV levels and the ECFV:ICFV ratio.

Biomarkers of cardiac stress, serum BNP and highsensitivity cardiac troponin levels as well as the number and class of antihypertensive medications used will be recorded at the mid-week HD session of weeks 1, 4, 12, 14 and 22 of the study. Diuretic use will not be recorded as participants with significant residual renal function are excluded.

Two types of survey will be administered and completed by the patient alone, or with the aid of the dialysis nurse. The first, provided at the beginning of each dialysis session, is a validated survey inquiring about the time it took the patient to recover to baseline following a previous dialysis session (interdialytic period) [48]. The second, provided at the end of each dialysis session, inquires about intradialytic symptoms of IDH, specifically nausea, vomiting, chest pain, shortness of breath, headache, muscle cramps, dizziness, fainting, fatigue and anxiety. A survey inquiring about nursing perception and attitudes of the BVM-guided UF biofeedback will be provided to the nurses at the end of the control and intervention phases.

\section{Sample size calculation}

We have previously estimated that $23 \%$ of our HD population have IDH [38]. To estimate the sample size, we conservatively assumed that only one IDH episode would occur per run (although it is likely that more than one episode will occur per run, increasing the study power). With this approach, and using a $2 \times 2$ crossover design, we estimated that a sample size of 30 participants would provide a power of $90 \%$ to demonstrate a $30 \%$ reduction in the rate of IDH under the biofeedback treatment vs. the control treatment, with a two-sided alpha of 0.05 . We will enroll 34 participants to guard against dropout, which can be as high as 30\% [41]. These estimates are based on simulation studies assuming only one IDH episode per run and two Poisson processes with average rates of IDH equal to 7 (control) and 5 (intervention) episodes over 24 runs. We used R (R Foundation for Statistical Computing, Vienna, Austria) [49] for simulations, and the package clusterPower in $\mathrm{R}$.

\section{Recruitment}

Recruitment will take place sequentially at the four participating HD units. From pilot data, we anticipate that approximately $20 \%$ of the $400 \mathrm{HD}$ patients in participating units will meet the criteria for IDH [38] and at least half of the eligible participants (40 participants) will be willing to participate in our study [50,51].

\section{Assignment of intervention, allocation concealment mechanism and implementation}

Randomization of allocation sequence will be done using computer-generated random numbers under the supervision of a statistician in the Department of Medicine, University of Calgary. Subsequently, the allocation sequence will be inserted into sequentially numbered, opaque, sealed envelopes. Following study consent and enrollment, an envelope containing the allocation sequence will be provided to the patient's HD nurse, who will implement the intervention according to the protocol.

\section{Blinding}

Given the nature of our intervention, and the inability to disable the BVM-guided UF biofeedback screens, prompts and alarms, we will not be able to blind the study personnel (KL) or the bedside dialysis nurse. The trial participants will be blinded to the intervention. To reduce bias, the HD nurse will be encouraged to document all intradialytic symptoms and interventions, as well as not to reveal the allocation sequence to the trial participant. In addition, study personnel (KL) will assess target weights and administer surveys uniformly. Study personnel involved with data abstraction and analysis will be blinded to the study intervention.

\section{Data collection}

\section{Collection of baseline and run-in phase data}

Baseline patient demographics (age, gender, HD vintage and race), comorbidities (e.g. congestive heart failure, 
diabetes mellitus and peripheral vascular disease), cause of renal disease, medications (number and class of antihypertensive), laboratory investigations (e.g. serum electrolytes, complete blood count and albumin), HD prescription (dialyzer type, composition, temperature, blood and dialysate flow rates, target weight, anticoagulation, use of sodium and/or UF profiles), and number of episodes of IDH over the preceding 8 weeks and run-in phase will be extracted from HD charts and local electronic health records by the study investigator.

\section{Collection of primary outcome variables}

Both sitting and standing blood pressure will be measured in a standardized fashion at the beginning and end of each dialysis session [52,53]. Intradialytic blood pressures will be measured in a sitting position every 30 minutes and at the time of IDH-related symptoms as defined in the outcomes section. Sitting blood pressures will be measured with the patient seated with their feet flat on the floor or a foot rest, back against a chair with a bare arm resting on a support, whereby the midpoint of the upper arm is at the level of the heart. Standing blood pressures will be measured with the patient standing feet flat on the floor, with a bare arm resting on a support, whereby the midpoint of the upper arm is at the level of the heart. An appropriatesized blood pressure cuff, where the cuff bladder length is $80 \%$ of the arm circumference, will be used. Blood pressure taken while the patient is in a Trendelenburg or supine position will be accepted if the patient is unable to sit during the pre-specified blood pressure measurement or is experiencing IDH-related symptoms. All blood pressures will be measured using an automated cuff attached to the dialysis machine. Manual blood pressure will be accepted if an automated blood pressure measurement cannot be obtained. Blood pressure, method of measurement, patient position, IDH-related symptoms and nursing interventions will be recorded by the bedside dialysis nurse.

\section{Collection of secondary outcome variables}

Dialysis adequacy, bioimpedance, BNP, high-sensitivity cardiac troponin, intra- and interdialytic symptom survey and antihypertensive medication use will be collected by study personnel at the pre-specified times (Table 1).

\section{Participant retention and follow-up}

Following enrollment, every effort will be made to follow up participants until the end of the study phase. Participants moving to different dialysis sites across the city of Calgary will be followed unless they move to a site not using the Fresenius 5008 machines, at which point they will be censored. Participants who move out of the city will be censored.

\section{Statistical analysis}

We will use mixed-effects Poisson regression to test the null hypothesis that the ratio of the rate of IDH episodes (main model exposure) during the intervention phase over the rate of IDH episodes during the control phase will range between 0.7 and 1.3 (i.e., the alternative twosided hypothesis is that the incidence rate ratio will be 0.7 or lower). We will study the effect of the intervention as a fixed effect and account for the correlation in the data due to the subject using random effects. The multi-level model will have errors due to within-subject variation and due to between-cluster variation (between subject variance). In the event that the study generates over-dispersed data, we will use a negative binomial regression with the approach as described above. We will

Table 1 Data collection schedule

\begin{tabular}{|c|c|c|c|c|c|c|c|c|c|}
\hline & \multirow[b]{2}{*}{$\begin{array}{l}\text { Every } \\
\text { session }\end{array}$} & \multicolumn{2}{|c|}{ Part 1} & & \multicolumn{2}{|c|}{ Part 2} & & \multicolumn{2}{|c|}{ Part 2} \\
\hline & & Mid-week 1 & Mid-week 4 & & Mid-week 8 & Mid-week 12 & & Mid-week 18 & Mid-week 22 \\
\hline Hemodialysis run sheet & $x$ & & & Randomization & & & Crossover and & & \\
\hline$K t / \mathrm{V}$ & $x$ & & & & & & washout & & \\
\hline Electrical bioimpedance & & $x$ & $x$ & & $x$ & $x$ & & $x$ & $x$ \\
\hline $\begin{array}{l}\text { Brain natriuretic } \\
\text { peptide }\end{array}$ & & $x$ & $x$ & & $x$ & $x$ & & $x$ & $x$ \\
\hline $\begin{array}{l}\text { High-sensitivity cardiac } \\
\text { troponin }\end{array}$ & & $x$ & $x$ & & $x$ & $x$ & & $x$ & $x$ \\
\hline $\begin{array}{l}\text { Intradialytic symptom } \\
\text { survey }\end{array}$ & $x$ & & & & & & & & \\
\hline $\begin{array}{l}\text { Interdialytic symptom } \\
\text { survey }\end{array}$ & $x$ & & & & & & & & \\
\hline Medication review & & $x$ & $x$ & & $x$ & $x$ & & $x$ & $x$ \\
\hline
\end{tabular}


also explore whether participants with larger drops in systolic blood pressure $(>30 \mathrm{mmHg})$ benefit from the planned intervention. They will be analyzed as a subgroup.

\section{Ethics}

Ethical approval has been granted from the University of Calgary Research Ethics Board. The research coordinator will determine patient eligibility, obtain consent from participants for participation in the study and access the sealed envelope containing allocation details. The coordinator will obtain the HD run sheets and submit them to an independent data entry clerk who is blinded to allocation. All data are kept strictly confidential. The principal investigator (JM) and study personnel (KL) are responsible for coordination of the study. The trial is registered with Clinicaltrials.gov (NCT01988181).

\section{Discussion}

Randomized studies assessing the role of BVM and BVM-guided UF biofeedback in the prevention of IDH are limited and of low quality. One the largest studies assessing BVM without biofeedback, which was done by Reddan et al., randomized 443 participants to BVM or conventional HD over a 6-month period. Participants in the BVM group had UF rates adjusted at the discretion of the bedside dialysis nurse with a suggested UF algorithm. Due to the need for manual adjustment of the UF rate, it is unclear if the nurses promptly responded to changes in relative blood volume using the suggested algorithm. In fact, the participants randomized to BVM had increased hospitalization and mortality, which may have reflected a lack of adherence to the UF algorithm, or alternatively could have been related to more cautious fluid removal due to the feedback provided by the BVM device [33].

However, when BVM is combined with biofeedback there may be a beneficial reduction in IDH and blood pressure $[31,32,41,54]$. In a 16 -week randomized crossover study by Dasselaar et al., 28 hypertensive patients were randomized to either BVM-guided UF and sodium biofeedback or standard HD for blood pressure and volume control. Despite a significant reduction in blood pressure of $22.5 / 8.3 \mathrm{~mm} \mathrm{Hg}$ in the BVM group and extracellular water to body volume ratios when compared to the standard HD group, the overall weight did not change. Although the values were not reported, this study also found a statistically significant reduction in dialysis hypotension [54]. A recent meta-analysis of six studies using BVM-guided biofeedback reported a 39\% overall reduction in the number of dialysis sessions complicated by IDH [55]. Of the six studies assessing IDH, three did not have IDH as the primary outcome. Five studies employed an intervention that included both dialysate sodium and UF biofeedback [31,34,56-58]. The combination of dialysate sodium and UF biofeedback over UF biofeedback alone raises concerns regarding the potential for sodium loading and resultant interdialytic weight gain through increased plasma osmolality and thirst, perpetuating the vicious cycle of IDH. In addition, the duration of the intervention phase in these studies ranged from 2 weeks to 6 months, with the majority using a short 2- to 4-week intervention, putting the long-term sustainability of the intervention into question. Three did not have a washout period between the intervention and control groups, and one did not have a run-in phase. The majority of the studies did not assess for changes in body fluid composition, nor did they address patient quality of life. In addition, the available studies are generally of low quality due to unclear randomization, unclear exclusion criteria and the inclusion of low numbers of IDH-prone participants [55].

Only one randomized crossover study (26 HD participants) examined the impact of BVM-guided UF biofeedback on patient symptoms during dialysis. The primary outcome was the percentage reduction in dialysis symptoms, which was $8 \%$. The secondary outcome was a reduction in hypotension [41]. The trial has several weaknesses including short duration (6 weeks), a lack of a washout period, a nonstandard definition of IDH and potential bias due to its industry-funded nature.

Currently, a prospective multi-center triple-arm parallelgroup crossover randomized controlled trial is in progress. It is comparing BVM-guided UF biofeedback and blood thermal monitoring temperature biofeedback, to BVMguided UF and sodium biofeedback, and to standard HD in fluid overloaded HD patients. The primary outcome in this study is the proportion of HD sessions that were complicated with intra- and post-dialytic symptoms related to UF, irrespective of blood pressure. The secondary outcomes include both symptomatic and asymptomatic IDH, using a non-standard definition of a $>40 \mathrm{~mm} \mathrm{Hg}$ drop in systolic blood pressure [59]. In contrast, our study focuses on enrolling symptomatic IDH patients, a standard IDH definition of $\geq 20 \mathrm{~mm} \mathrm{Hg}$, and studying only BVM-guided UF biofeedback without another accompanying biofeedback technology.

We have described our methods for recruitment, randomization, allocation concealment, dialysis intervention, outcome assessment and data collection methods in detail. The study protocol was developed according to the Standard Protocol Items: Recommendations for Interventional Trials (SPIRIT) 2013 [60]. It will be conducted and the results reported following the Consolidated Standards of Reporting Trials (CONSORT) statement [35].

\section{Trial status}

Enrollment began in June 2014 with an expected completion date of July 2015. 


\section{Additional file}

Additional file 1: Nursing IDH Management Algorithm. This is the current local protocol for management of IDH that will be followed by the nurses.

\section{Abbreviations}

BNP: brain natriuretic peptide; BVM: blood volume monitoring; CONSORT: Consolidated Standards of Reporting Trials; DRIP: dry-weight reduction in hypertensive hemodialysis patient; ECFV: extracellular fluid volume; HD: hemodialysis; ICFV: intracellular fluid volume; IDH: intradialytic hypotension; SPIRIT: Standard Protocol Items: Recommendations for Interventional Trials; UF: ultrafiltration.

\section{Competing interests}

The authors declare that they have no competing interests.

\section{Authors' contributions}

$\mathrm{KL}$ conceived and designed the study, collected and analyzed the data, wrote the manuscript and gave final approval for the manuscript. JM and RQ conceived and designed the study, collected and analyzed the data, made critical revisions and gave final approval for the manuscript. PR collected and analyzed the data, made critical revisions and gave final approval for the manuscript. All authors read this manuscript; they approve of its publication and agree to be accountable for the accuracy and integrity of the work.

\section{Acknowledgements}

The authors thank the Roy and Vi Baay Foundation through the University of Calgary for sponsoring the research. The funding agency had no role in the development of the study design, data collection process or the preparation of the manuscript for publication.

\section{Author details}

'Department of Medicine, University of Calgary, Calgary, Canada.

${ }^{2}$ Department of Community Health Sciences, University of Calgary, Calgary, Canada.

Received: 21 March 2014 Accepted: 2 December 2014 Published: 10 December 2014

\section{References}

1. Renal Data System US: USRDS 2012 Annual Data Report: Atlas of Chronic Kidney Disease and End-Stage Renal Disease in the United States. Bethesda MD: 2012

2. US Renal Data System: USRDS 2011 Annual Data Report: Atlas of End-Stage Renal Disease in the United States. Bethesda, MD: 2011:183-194.

3. Canadian Institute for Health Information: Treatment of End-Stage Organ Failure in Canada 2001-2010; 2012.

4. Mailloux LU, Bellucci AG, Wilkes BM, Napolitano B, Mossey RT, Lesser M, Bluestone PA: Mortality in dialysis patients: analysis of the causes of death. Am J Kidney Dis 1991, 18:326-335.

5. Wizemann V, Wabel P, Chamney P, Zaluska W, Moissl U, Rode C, Malecka-Masalska T, Marcelli D: The mortality risk of overhydration in haemodialysis patients. Nephrol Dial Transplant 2009, 24:1574-1579.

6. Kalantar-Zadeh $\mathrm{K}$, Regidor DL, Kovesdy CP, Van Wyck D, Bunnapradist S, Horwich TB, Fonarow GC: Fluid retention is associated with cardiovascular mortality in patients undergoing long-term hemodialysis. Circulation 2009, 119:671-679.

7. Velasco N, Chamney P, Wabel P, Moissl U, Imtiaz T, Spalding E, McGregor M, Innes A, MacKay I, Patel R, Jardine A: Optimal fluid control can normalize cardiovascular risk markers and limit left ventricular hypertrophy in thrice weekly dialysis patients. Hemodial Int 2012, 16:465-472.

8. Henrich WL: Hemodynamic instability during hemodialysis. Kidney Int 1986, 30:605-612.

9. Tattersall J, Martin-Malo A, Pedrini L, Basci A, Canaud B, Fouque D, Haage $P$, Konner K, Kooman J, Pizzarelli F, Tordoir J, Vennegoor M, Wanner C, ter Wee P, Vanholder R: EBPG guideline on haemodynamic instability. Nephrol Dial Transplant 2007, 22 Suppl 2:ii22-ii44.
10. National Kidney Foundation: K/DOQI clinical practice guidelines for cardiovascular disease in dialysis patients. Am J Kidney Dis 2005, 45(4 Suppl 3):S1-S153.

11. Schreiber MJ: Setting the stage. Am J Kidney Dis 2001, 38(4 Suppl 4):S1-S10.

12. Zager PG, Nikolic J, Brown RH, Campbell MA, Hunt WC, Peterson D, Van Stone J, Levey A, Meyer KB, Klag MJ, Johnson HK, Clark E, Sadler JH, Teredesai P: ' $U$ ' curve association of blood pressure and mortality in hemodialysis patients. Kidney Int 1998, 54:561-569.

13. Shoji T, Tsubakihara Y, Fujii M, Imai E: Hemodialysis-associated hypotension as an independent risk factor for two-year mortality in hemodialysis patients. Kidney Int 2004, 66:1212-1220.

14. Yoshimitsu T, Hirakata H, Fujii K, Kanai H, Hirakata E, Higashi H, Kubo M, Tanaka H, Shinozaki M, Katafuchi R, Yokomizo Y, Oh Y, Tomooka S, Fujimi S, Fujishima M: Cerebral ischemia as a causative mechanism for rapid progression of brain atrophy in chronic hemodialysis patients. Clin Nephrol 2000, 53:445-451.

15. Ishida I, Hirakata H, Sugimori H, Omae T, Hirakata E, Ibayashi S, Kubo M, Fujishima M: Hemodialysis causes severe orthostatic reduction in cerebral blood flow velocity in diabetic patients. Am J Kidney Dis 1999, 34:1096-1104.

16. Breidthardt T, Burton JO, Odudu A, Eldehni MT, Jefferies HJ, Mclntyre CW: Troponin T for the detection of dialysis-induced myocardial stunning in hemodialysis patients. Clin J Am Soc Nephrol 2012, 7:1285-1292.

17. Burton JO, Jefferies HJ, Selby NM, Mclntyre CW: Hemodialysis-induced cardiac injury: determinants and associated outcomes. Clin J Am Soc Nephrol 2009, 4:914-920.

18. Burton JO, Jefferies HJ, Selby NM, McIntyre CW: Hemodialysis-induced repetitive myocardial injury results in global and segmental reduction in systolic cardiac function. Clin J Am Soc Nephrol 2009, 4:1925-1931.

19. Selby NM, Mclntyre CW: The acute cardiac effects of dialysis. Semin Dial 2007, 20:220-228

20. Burton JO, Korsheed S, Grundy BJ, McIntyre CW: Hemodialysis-induced left ventricular dysfunction is associated with an increase in ventricular arrhythmias. Ren Fail 2008, 30:701-709.

21. Johner C, Chamney PW, Schneditz D, Krämer M: Evaluation of an ultrasonic blood volume monitor. Nephrol Dial Transplant 1998, 13:2098-2103.

22. Mann H, Stiller S, Schallenberg U, Thömmes A: Optimizing dialysis by variation of ultrafiltration rate and sodium concentration controlled by continuous measurement of circulating blood volume. Contrib Nephrol 1989, 74:182-190.

23. Mancini E, Santoro A, Spongano M, Paolini F, Rossi M, Zucchelli P: Continuous on-line optical absorbance recording of blood volume changes during hemodialysis. Artif Organs 1993, 17:691-694.

24. Dasselaar JJ, Huisman RM, DE Jong PE, Franssen CFM: Relative blood volume measurements during hemodialysis: comparisons between three noninvasive devices. Hemodial Int 2007, 11:448-455.

25. De Vries JP, Kouw PM, van der Meer NJ, Olthof CG, Oe LP, Donker AJM, de Vries PM: Non-invasive monitoring of blood volume during hemodialysis: its relation with post-dialytic dry weight. Kidney Int 1993, 44:851-854.

26. Booth J, Pinney J, Davenport A: Do changes in relative blood volume monitoring correlate to hemodialysis-associated hypotension? Nephron Clin Pr 2011, 117:c179-c183.

27. Agarwal R, Kelley K, Light RP: Diagnostic utility of blood volume monitoring in hemodialysis patients. Am J Kidney Dis 2008, 51:242-254.

28. Sinha AD, Light RP, Agarwal R: Relative plasma volume monitoring during hemodialysis aids the assessment of dry weight. Hypertension 2010, 55:305-311.

29. Krepel HP, Nette RW, Akçahüseyin E, Weimar W, Zietse R: Variability of relative blood volume during haemodialysis. Nephrol Dial Transplant 2000, 15:673-679

30. Steuer RR, Leypoldt JK, Cheung AK, Senekjian HO, Conis JM: Reducing symptoms during hemodialysis by continuously monitoring the hematocrit. Am J Kidney Dis 1996, 27:525-532.

31. Santoro A, Mancini E, Basile C, Amoroso L, Di Giulio S, Usberti M, Colasanti G, Verzetti G, Rocco A, Imbasciati E, Panzetta G, Bolzani R, Grandi F, Polacchini M: Blood volume controlled hemodialysis in hypotension-prone patients: a randomized, multicenter controlled trial. Kidney Int 2002, 62:1034-1045.

32. Ronco C, Brendolan A, Milan M, Rodeghiero MP, Zanella M, La Greca G: Impact of biofeedback-induced cardiovascular stability on hemodialysis tolerance and efficiency. Kidney Int 2000, 58:800-808.

33. Reddan DN, Szczech LA, Hasselblad V, Lowrie EG, Lindsay RM, Himmelfarb J, Toto RD, Stivelman J, Winchester JF, Zillman LA, Califf RM, Owen WF 
Intradialytic blood volume monitoring in ambulatory hemodialysis patients: a randomized trial. J Am Soc Nephrol 2005, 16:2162-2169.

34. Nesrallah GE, Suri RS, Thiessen-Philbrook H, Heidenheim P, Lindsay RM: Can extracellular fluid volume expansion in hemodialysis patients be safely reduced using the hemocontrol biofeedback algorithm? A randomized trial. ASAIO J 2008, 54:270-274.

35. Schulz KF, Altman DG, Moher D: CONSORT 2010 Statement: updated guidelines for reporting parallel group randomised trials. Trials 2010, 11:32.

36. Penne EL, Sergeyeva O: Sodium gradient: a tool to individualize dialysate sodium prescription in chronic hemodialysis patients? Blood Purif 2011, 31:86-91.

37. Agarwal R, Alborzi P, Satyan S, Light RP: Dry-weight reduction in hypertensive hemodialysis patients (DRIP): a randomized, controlled trial. Hypertension 2009, 53:500-507.

38. Tai DJ, Conley J, Ravani P, Hemmelgarn BR, MacRae JM: Hemodialysis prescription education decreases intradialytic hypotension. J Nephrol 2012, 26:315-322.

39. Schneditz D, Pogglitsch $H$, Horina J, Binswanger U: A blood protein monitor for the continuous measurement of blood volume changes during hemodialysis. Kidney Int 1990, 38:342-346.

40. Automated blood volume regulation during hemodialysis. In [http://cdn intechopen.com/pdfs/21270/InTech-Automated_blood_volume_regulation_ during_hemodialysis.pdf

41. Gabrielli D, Krystal B, Katzarski K: Improved intradialytic stability during haemodialysis with blood volume-controlled ultrafiltration. J Nephrol 2008, c:232-240.

42. Kuhlmann U, Goldau R, Samadi N, Graf T, Gross M, Orlandini G, Lange H: Accuracy and safety of online clearance monitoring based on conductivity variation. Nephrol Dial Transplant 2001, 16:1053-1058.

43. Lukaski HC, Johnson PE, Bolonchuk WW, Lykken Gl: Assessment of fat-free mass using bioelectrical impedance measurements of the human body. Am J Clin Nutr 1985, 41:810-817.

44. Chan C, McIntyre C, Smith D, Spanel P, Davies SJ: Combining near-subject absolute and relative measures of longitudinal hydration in hemodialysis. Clin J Am Soc Nephrol 2009, 4:1791-1798.

45. Chamney PW, Wabel P, Moissl UM, Müller MJ, Bosy-Westphal A, Korth O, Fuller NJ: A whole-body model to distinguish excess fluid from the hydration of major body tissues. Am J Clin Nutr 2007, 85:80-89.

46. Wabel P, Chamney P, Moissl U, Jirka T: Importance of whole-body bioimpedance spectroscopy for the management of fluid balance. Blood Purif 2009, 27:75-80.

47. Wabel P, Moissl U, Chamney P, Jirka T, Machek P, Ponce P, Taborsky P, Tetta C, Velasco N, Vlasak J, Zaluska W, Wizemann V: Towards improved cardiovascular management: the necessity of combining blood pressure and fluid overload. Nephrol Dial Transplant 2008, 23:2965-2971.

48. Lindsay RM, Heidenheim PA, Nesrallah G, Garg AX, Suri R: Minutes to recovery after a hemodialysis session: a simple health-related quality of life question that is reliable, valid, and sensitive to change. Clin J Am Soc Nephrol 2006, 1:952-959.

49. R Foundation for Statistical Computing. In [http://www.R-project.org/]

50. MacRae JM, Ahmed SB, Atkar R, Hemmelgarn BR: A randomized trial comparing buttonhole with rope ladder needling in conventional hemodialysis patients. Clin J Am Soc Nephrol 2012, 7:1632-1638.

51. Tai DJ, Ahmed SB, Palacios-Derflingher L, Hemmelgarn BR, MacRae JM: Pneumatic compression devices during hemodialysis: a randomized crossover trial. Nephrol Dial Transplant 2013, 28:982-990.

52. Program CHE: The 2013 Canadian Hypertension Education Program Recommendations. Markham, ON Canada: 2013.

53. Reeves RA: The rational clinical examination. Does this patient have hypertension? How to measure blood pressure. JAMA 1995, 273:1211-1218.

54. Dasselaar JJ, Huisman RM, de Jong PE, Burgerhof JGM, Franssen CFM: Effects of relative blood volume-controlled hemodialysis on blood pressure and volume status in hypertensive patients. ASAIO J 2007, 53:357-364

55. Nesrallah GE, Suri RS, Guyatt G, Mustafa RA, Walter SD, Lindsay RM, Akl EA: Biofeedback dialysis for hypotension and hypervolemia: a systematic review and meta-analysis. Nephrol Dial Transplant 2013, 28:182-191.

56. Bégin V, Déziel C, Madore F: Biofeedback regulation of ultrafiltration and dialysate conductivity for the prevention of hypotension during hemodialysis. ASAIO J 2002, 48:312-315
57. Déziel C, Bouchard J, Zellweger M, Madore F: Impact of hemocontrol on hypertension, nursing interventions, and quality of life: a randomized, controlled trial. Clin J Am Soc Nephrol 2007, 2:661-668.

58. Selby NM, Lambie SH, Camici PG, Baker CS, Mclntyre CW: Occurrence of regional left ventricular dysfunction in patients undergoing standard and biofeedback dialysis. Am J Kidney Dis 2006, 47:830-841.

59. Hecking M, Antlanger M, Winnicki W, Reiter T, Werzowa J, Haidinger M, Weichhart T, Polaschegg H-D, Josten P, Exner I, Lorenz-Turnheim K, Eigner M, Paul G, Klauser-Braun R, Hörl WH, Sunder-Plassmann G, Säemann MD: Blood volume-monitored regulation of ultrafiltration in fluidoverloaded hemodialysis patients: study protocol for a randomized controlled trial. Trials 2012, 13:79.

60. Chan A, Tetzlaff JM, Altman DG, Dickersin K, Moher D: SPIRIT 2013: new guidance for content of clinical trial protocols. Lancet 2013, 381:91-92.

\section{doi:10.1186/1745-6215-15-483}

Cite this article as: Leung et al:: Ultrafiltration biofeedback guided by blood volume monitoring to reduce intradialytic hypotensive episodes in hemodialysis: study protocol for a randomized controlled trial. Trials 2014 15:483.

\section{Submit your next manuscript to BioMed Central and take full advantage of:}

- Convenient online submission

- Thorough peer review

- No space constraints or color figure charges

- Immediate publication on acceptance

- Inclusion in PubMed, CAS, Scopus and Google Scholar

- Research which is freely available for redistribution 